\title{
ANALYSIS OF PRESSURE BLIPS IN AFT-FINOCYL SOLID ROCKET MOTOR
}

\author{
M. Di Giacinto, B. Favini, and E. Cavallini \\ Sapienza University of Rome \\ Department of Mechanical and Aerospace Engineering \\ 18 Via Eudossiana, Rome I-00184, Italy
}

\begin{abstract}
Ballistic anomalies have frequently occurred during the firing of several solid rocket motors (SRMs) (Inertial Upper Stage, Space Shuttle Redesigned SRM (RSRM) and Titan IV SRM Upgrade (SRMU)), producing even relevant and unexpected variations of the SRM pressure trace from its nominal profile. This paper has the purpose to provide a numerical analysis of the following possible causes of ballistic anomalies in SRMs: an inert object discharge, a slag ejection, and an unexpected increase in the propellant burning rate or in the combustion surface. The SRM configuration under investigation is an aft-finocyl SRM with a first-stage/small booster design. The numerical simulations are performed with a quasi-one-dimensional (Q1D) unsteady model of the SRM internal ballistics, properly tailored to model each possible cause of the ballistic anomalies. The results have shown that a classification based on the head-end pressure (HEP) signature, relating each other the HEP shape and the ballistic anomaly cause, can be made. For each cause of ballistic anomalies, a deepened discussion of the parameters driving the HEP signatures is provided, as well as qualitative and quantitative assessments of the resultant pressure signals.
\end{abstract}

\section{INTRODUCTION}

Ballistic anomalies in SRMs may produce relevant and unexpected variations of the motor pressure trace with the respect to its nominal and predicted behavior. These pressure disturbances, also referred to as blips in the literature, as a consequence, may determine relevant and important modifications of the thrust delivered by the SRM. The ballistic anomalies can last a time period from few milliseconds to some seconds/tens of seconds, resulting, in the first case, only in a temporary modification of the pressure trace of the motor, or, in the second case, in a complete and relevant deviation of the SRM behavior, 
from their occurrence up to the burnout. Moreover, in rare cases, the cause of the ballistics anomaly may bring about the complete failure of the SRM itself.

In this work, a review of possible causes for the occurrence of the ballistic anomalies will be presented and discussed in detail providing a classification of the produced pressure blips as related to each possible root cause event. A numerical study will be performed for the different possible causes of a pressure blip, considering as reference configuration an aft-finocyl SRM with a first stage or booster design, charged with an aluminized propellant. Each possible cause of the ballistics anomalies will be investigated by means of a parametric analysis with the use of an unsteady Q1D model of the SRM internal ballistic, properly tailored to represent each possible cause of the pressure blips. The aim is to have qualitative and quantitative comparisons among the possible different chamber responses in order to outline a relationship among different shapes of the pressure blips and the possible different root causes of the ballistic anomalies. In particular, the attention will be focused on ballistic anomalies causing a temporary modification of the nominal pressure trace of the SRM rather than to those causing a complete drift of the SRM behavior.

In general, the main causes of ballistic anomalies in SRMs are the following:

(1) a mass ejection, resulting from the detachment of an object from inner components of the SRMs;

(2) a slag ejection phenomenon;

(3) the occurrence of high burning rate pockets of propellant;

(4) an unexpected increase in the burning surface area; and

(5) an effect of combustion instability.

The last cause, the dynamic combustion effect, even if of wide interest for basic research activities, will not be considered in this work, since usually modern SRMs are intentionally designed to not be affected by combustion instability problems.

This paper will be structured as follows. First, the characteristics of each of the possible causes of the ballistics anomalies will be briefly discussed by performing a literature survey in section 2 , which also describes some real cases in which ballistics anomalies occurred. Then, the models used for the simulation of the root causes of the ballistic anomalies will be described in section 3, discussing for each cause the way in which it has been modeled. After that, the reference SRM configuration considered in this study will be depicted in section 4 and, finally, the results of each possible cause of the ballistic anomalies will be discussed in section 5 . 


\section{LITERATURE SURVEY ON BALLISTIC ANOMALIES IN SOLID ROCKET MOTORS}

Three main root causes can be identified in the literature studies that discussed and analyzed ballistic anomalies in SRMs. A similar classification of them was provided also in [1], from which this literature survey has taken some inspiration.

The first one is the discharge of an inert body that was considered responsible for the failure of the second-stage SRM of the Inertial Upper Stage (IUS) in the spring of 1983, as discussed in [2] and one of the proposed causes for the pressure and thrust anomaly occurred during the firing of the first stage of the IUS motor, fired in November 1979, as analyzed in [1] (in particular, the objects were identified as the igniter, its aluminum housing, and internal insulation). In [2], in order to reconstruct the experimental thrust anomaly, Murdock simulated the expulsion of single-multiple inert masses with flat-faced cylindrical shape and different dimensions that obstructed the nozzle throat area with an unsteady axisymmetric model.

The second one is a slag ejection phenomenon, which caused ballistics anomalies of Space Shuttle RSRM in the flight STS-54 (RSRM-29) on January 13, 1993 and of the Titan IV SRMU in several flights. This anomaly was deeply analyzed by several investigators with both analytical/numerical models and small-scale cold firing tests and full-scale hot firing tests as reported in [3-7]. The slag ejection phenomenon is related to the transit of a continuous stream of slag of alumina through the nozzle. This phenomenon may occur at certain times into the firing because of the detachment of relevant parts of slag from the slag pool, located in the submergence region and at the nozzle back-face as a consequence of the nozzle vectoring or the acceleration environment to which the SRM is subjected during the firing.

The third one is an unexpected sudden increase in the burning rate or in the combustion surface (due to voids in the grain propellant compound or cracks and debonds), as argued by Heister and Landsbaum in [1], or a dynamic burning effect which may cause pressure spikes during the quasi-steady state of SRMs, as discussed for tactical motors by Blomshield in [8].

The first two root causes for the ballistic anomalies (discharge of an inert object and slag ejection), while inferring different phenomena occurred in the chamber, consider the same intermediate effect, which, in turn, was responsible for anomalous SRM internal ballistic and performance: a temporary and sudden nozzle throat obstruction by "something coming from the inner parts of the SRM." Anyhow, they need to be discussed separately, since they describe different root causes that cannot be treated in the same manner. For the IUS SRM, it is more or less generically, depending on the author, the expulsion of an inert mass from the nozzle, an igniter that has come loose, for [2], or the igniter, its insulation and housing, for [1]. Instead, for the Space Shuttle RSRM and 
the Titan IV SRMU, the cause is the sudden expulsion of a continuous stream of molten aluminum oxide coming from the slag pool which accumulates in the submergence region of such kind of SRMs because of the combination of the submersed nozzle design and the aluminized propellant formulation [3,5-7]. Even though both the cases consider the same intermediate effect on the SRM internal ballistics (a temporary obstruction of the nozzle throat), the two phenomena have to be modeled in different manner, as also done in the related references, considering that the passage of an object advected in the nozzle flow and the expulsion of a continuous stream of slag are very different phenomena that can have different characteristic times of occurrence and, therefore, different effects on the SRM internal ballistic.

The third class of possible causes, instead, is related to combustion phenomena occurring at the grain propellant surface:

(1) a sudden propellant burning rate anomaly, such as a "pocket of propellant which burns at explosive or even near detonation velocities," as argued by Heister and Landsbaum in [1] for the IUS SRM-2;

(2) a sudden increase of the propellant grain burning surface, as discussed in [1], due to the possible presence of voids, cracks, or debonds in the propellant grain; and

(3) a dynamic response of the propellant grain, which may cause the onset of self-sustained (in some cases, bringing to catastrophic failures), or suddenly damped pressure perturbations inside the combustion chamber, as widely discussed in the relevant literature (among all the papers, a very good discussion of the problem with a wide database of experimental tests in the one of Ref. [8]).

The study of particular cases, involving a combination of the ejection of inert body from the nozzle and the consequent triggering of a dynamic combustion response is discussed in the work [9], for a subscale laboratory SRM.

\section{SOLID ROCKET MOTOR INTERNAL FLOWFIELD MODEL FOR BALLISTIC ANOMALIES INVESTIGATION}

The model considered for the simulation of all the ballistics anomalies is a Q1D unsteady model of the SRM internal ballistics, properly adapted to account for the different possible root causes of the ballistic anomalies. The model is a derivation of the SPIT model [10-13] developed for a study of the fluid/structural interaction during SRM ignition transient, presented in [14], able to account for 
the variations in space and time of the SRM internal geometry. The governing equations are the classical Euler set of partial differential equations with the addition of proper source terms in order to account for the presence of cavities (slots, submergence region and floaters), propellant grain mass and energy addition into the flow, with the addition, in case, of the coupling terms due to objects motion in the flow:

$$
\begin{gathered}
\frac{\partial\left(\rho A_{p}\right)}{\partial t}+\frac{\partial\left(\rho u A_{p}\right)}{\partial x}=\underbrace{r_{b} P_{b} \rho_{p}}_{\text {propellant }}+\underbrace{\frac{\dot{m}_{s} A_{p}}{V}}_{\text {cavities }} ; \\
\frac{\partial\left(\rho u A_{p}\right)}{\partial t}+\frac{\partial\left[\left(\rho u^{2}+p\right) A_{p}\right]}{\partial x}-p \frac{\partial A_{p}}{\partial x}=\underbrace{\frac{1}{2} \rho u^{2} c_{f}}_{\text {friction }}+\underbrace{\frac{\left(F_{D}\right) A_{p}}{V}}_{\text {particle }} ; \\
\frac{\partial\left(\rho e A_{p}\right)}{\partial t}+\frac{\partial\left[(\rho e+p) u A_{p}\right]}{\partial x}=-p \frac{\partial A_{p}}{\partial t}+\underbrace{r_{b} P_{b} \rho_{p} H_{f}}_{\text {propellant }}+\underbrace{\frac{\dot{m}_{s} A_{p} H_{s}}{V}}_{\text {cavities }} .
\end{gathered}
$$

Each of the possible causes of the ballistic anomalies presented in the previous sections is then modeled and coupled with the Q1D model, as will be described in the following.

\subsection{Ejection of Inert Object}

In order to consider the simulation of an object advected in the flowfield, the basic hypothesis is that it can be assumed as an inert particle, locally in thermal equilibrium with the surrounding flowfield, so that no energy exchange occurs among the flowfield and the particle itself. As a consequence, the effects of the particle on the carrier flow are only related to the momentum equation because of drag force acting on the particle itself and volume effects, because particle volume is not negligible. A further simplification is that the particle is a sphere with an imposed radius:

$$
\left.\begin{array}{c}
\frac{d x_{p}}{d t}=v_{p} ; \quad \operatorname{Re}_{p}=\frac{\rho\left|u\left(x_{p}\right)-v_{p}\right| d_{p}}{\mu} ; \quad M_{p}=\frac{\left|u\left(x_{p}\right)-v_{p}\right|}{\sqrt{\gamma R T}} ; \\
\frac{d v_{p}}{d t}=\frac{u\left(x_{p}\right)-v_{p}}{\tau_{p}} ; \quad \tau_{p}=\frac{4 \rho_{p} d_{p}}{3 C_{D}\left|u\left(x_{p}\right)-v_{p}\right|} .
\end{array}\right\}
$$

Under such hypotheses, the motion of the particle can be expressed as given by Eqs. (1), using the classical set of Lagrangian equations for a droplet in a high Reynolds flowfield. 


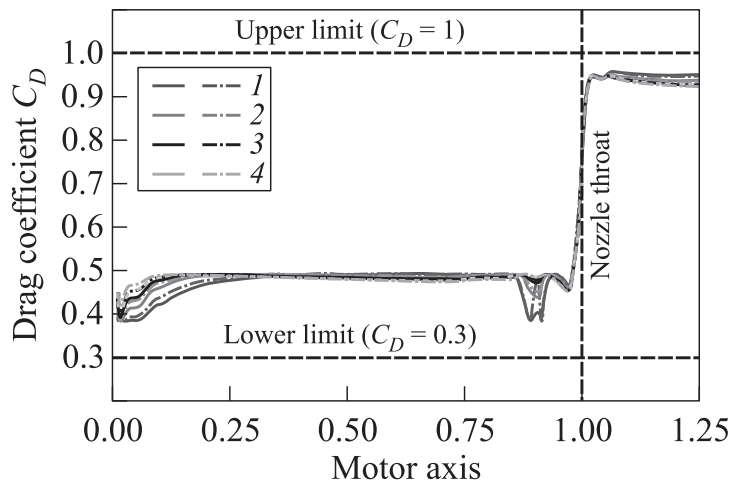

Figure 1 Drag coefficient envelope definition: $1-r_{p}=25 \mathrm{~mm} ; 2-50 ; 3-75$; and $4-r_{p}=100 \mathrm{~mm}$. Solid curves refer to $p=67$ bar and dash-dotted curves refer to $p=82$ bar

In Eqs. (1), only the drag term is considered, since virtual mass force, lift force, and buoyancy force become negligible for the problem under investigation (big and heavy particles, $\rho_{p} / \rho \gg 1$ ).

For the numerical simulations, the identification of an upper and lower limit for the drag coefficient and for the particle density is necessary in order to perform a parametric analysis able to give both qualitative and quantitative assessments, with a reasonable margin of uncertainty, of the effects on the SRM internal ballistics of particle motion within the flowfield. The uncertainties are primarily related to the aerodynamics of the object carried within the SRM flowfield, for the determination of the force acting on the object itself, which is strongly variable between an almost incompressible regime, far from the nozzle entrance, and compressible regime, inside the nozzle. Secondarily, some uncertainties to be considered are related to the density of the object detached from the internal components of the SRM.

For the drag coefficient $\left(C_{D}\right.$ in Eqs. (1)) evaluation and, in turn, to assess the drag force $(D)$ acting on the droplet, a strong deviation from the situation $\operatorname{Re}_{p}=1$ and $M_{p} \ll 1$ is expected to occur for the flowfield conditions inside the SRM and, therefore, a correction to the classical Stokes solution has to be considered. This is done employing the expression of the compressibility/high Reynolds correction provided in [15] that is a modification of the Clift-Gauvin drag coefficient expression [16]. Moreover, the upper and lower limits selected for the drag coefficient in Fig. 1 are taken into account, since the correlations used for the drag coefficient stand in uniform flowfield conditions upstream and downstream the particle itself and before establishing of the supercritical regime of the particle motion where a strong drop of the drag coefficient occurs for very high Reynolds numbers. In fact, a uni- 
form flowfield for the particle motion inside the SRM stands up to around the nozzle entrance, but not within the nozzle, where the flowfield is strongly nonuniform because of the abrupt expansion and acceleration of the flow. Furthermore, the supercritical regime and the compressibility effects may characterize the motion of the particle within the nozzle, especially for big particles.

As far as the particle density is concerned, the following upper and lower limits will be considered: $\rho_{p}=1500 \mathrm{~kg} / \mathrm{m}^{3}$, representative the density of a carbonphenolic thermal protection, and $\rho_{p}=1900 \mathrm{~kg} / \mathrm{m}^{3}$, representative the density of a carbon-carbon thermal protection. Note that this interval contains also the density of the propellant grain, which is around $1700-1800 \mathrm{~kg} / \mathrm{m}^{3}$ and, also, the density of the alumina particles at the temperature inside the SRM $\left(\rho_{\mathrm{Al}_{2} \mathrm{O}_{3}} \approx 1600 \mathrm{~kg} / \mathrm{m}^{3}[17]\right)$, considering valid the JANNAF (Joint Army Navy NASA Air Force) extrapolation for the alumina density and the experimental data extrapolated from Titan IV and SICB SRMs [17], as well as the density of a high-strength composite material which constitute, for example, the casing of an expendable igniter.

It is worth underlining that for the modeling approach chosen for the phenomenon (Q1D), no assessment or parametric analysis will be made regarding the possibility that a near-spherical particle, in a given simulation setup (drag coefficient, density, radius, etc.), can remain entrapped somewhere inside the SRM, or hit the nozzle nose.

Validation against experimental data of a small-scale solid rocket motor. A validation of the model proposed for the particle motion within the SRM flowfield has been successfully performed against the experimental data provided by Lovine et al. in [9], for a small-scale laboratory SRM of cylindrical shape, in which the particle transit in the SRM flowfield was used in order to trigger the combustion instability of the SRM. Since no model for the dynamic burning effects is implemented in this work, the comparison has been limited to the first strokes caused by the particle transit in the flow. Looking at the comparison between the numerical simulations and the experimental data in Fig. 2, in all cases, a very good matching with the experimental data is obtained in terms of shape and amplitude of the pressure signals, especially for the pressure at the nozzle entrance section (aft-end in Fig. 2), whereas the pressure traces at the head-end of the SRM show a less good quantitative agreement with experimental data. Nevertheless, a general very good qualitative agreement between the numerical simulations and the experimental data can be appreciated as well as a very good matching with the experimental data in terms of shape of the strokes and their duration in time.

The numerical strokes of the HEP show always around the same differences with respect to the experimental data: a slight early occurrence in time and a slight underestimation of the peak of amplitude of the stroke. The first point 


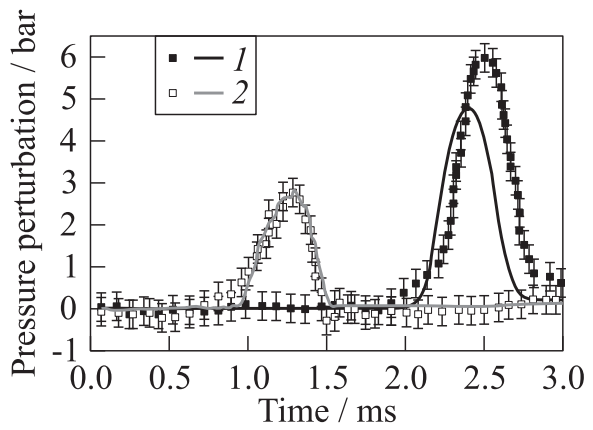

(a)

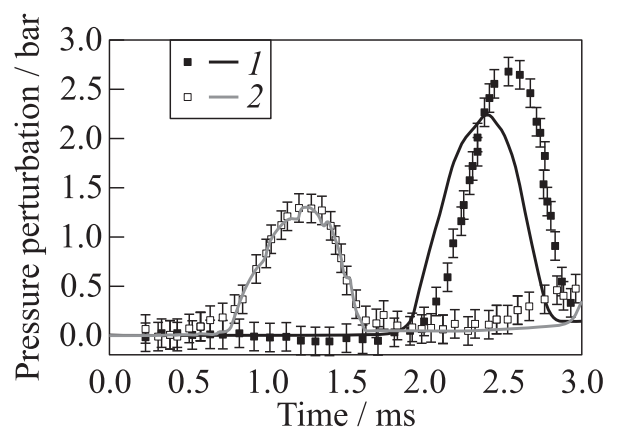

(b)

Figure 2 Comparison between numerical simulations (curves) and experimental data (signs) at fore-end (1) and aft-end (2) taken from [9]: $(a)$ test 5 - pulse 2: nylon droplet of diameter $15.24 \mathrm{~mm}$ at $\approx 60$ bar; and $(b)$ test 7 - pulse 1: steel droplet of diameter $11.1125 \mathrm{~mm}$ at $\approx 52$ bar

is directly due to a nonperfect reconstruction of the experimental test case described in [9]. In fact, the slight early occurrence of the head-end stroke is directly due to an approximate reconstruction of the laboratory-scale acoustics that can be ascribable to one or the combination of the following uncertainties on the motor: the chamber head-to-throat length (which cannot be extracted, if not in a approximate manner, from the description of the SRM); the propellant grain properties in terms of adiabatic flame temperature, specific heat ratio, and molecular weight (the propellant is generally defined as an ammonium perchlorate (AP) / hydroxyl-terminated polybutadiene (HTPB) propellant without any other reference to its detailed characteristics); and the mutual distance among the pressure gauges located at the fore-end and aft-end (nozzle entrance section). Instead, the slight underestimation of the amplitude of the HEP strokes is supposed to be due to the missing in the simulation model of the dynamic combustion response of the propellant which may have been triggered by the pressure stroke generated by the transit of the particle in the SRM internal flowfield.

\subsection{Slag Ejection}

The slag ejection phenomenon, in principle, represents a very difficult event to be modeled, since it involves the need of a characterization of several and complex phenomena: the slag pool generation during the firing (which implies to model the alumina particles entrapment mechanism within the submergence region); the simulation of the cause of the detachment from the slag pool of a flow of 


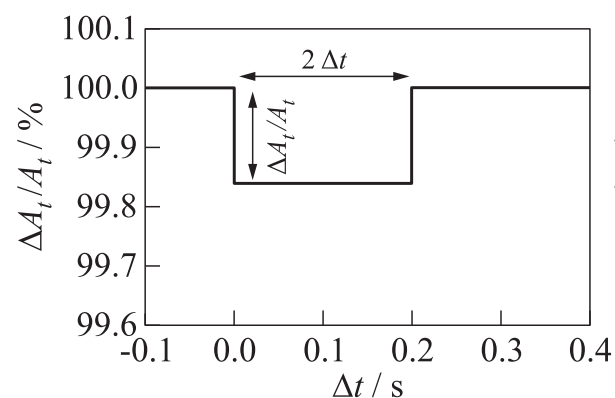

(a)

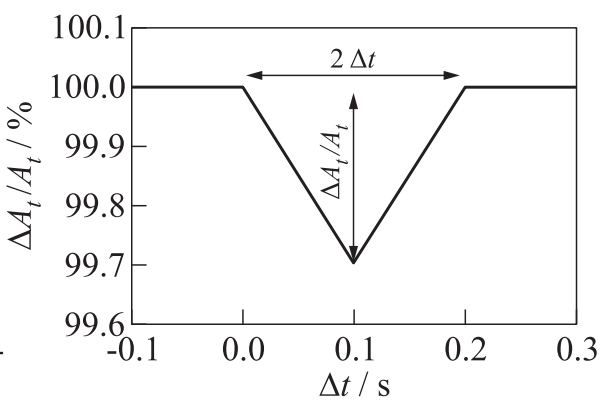

(b)

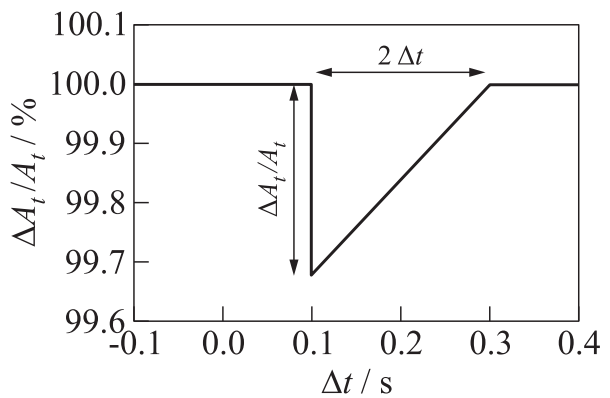

(c)

Figure 3 Imposed laws of nozzle throat obstruction due to slag ejection $(\Delta t$ $=100 \mathrm{~ms}):(a)$ rectangular law $\left(\Delta A_{t} / A_{t}=0.16 \%\right) ;(b)$ triangular law $\left(\Delta A_{t} / A_{t}\right.$ $=0.3 \%)$; and $(c)$ saw-tooth law $\left(\Delta A_{t} / A_{t}=0.328 \%\right)$

alumina; the characterization of the flow of alumina within the SRM flowfield and, finally, its effects on the SRM internal ballistic.

In this work, the approach used is a very simplified one, also common to the approaches used by the authors who investigated this phenomenon in the literature (refer to section 2). The idea is to model the slag ejection through only its principal source of perturbation of the SRM internal ballistic: the obstruction of the nozzle throat available to the gas flow. Hence, different kinds of nozzle throat variations in time have been imposed to the SRM during the simulation in order to characterize the slag ejection phenomenon: rectangular, triangular, and saw-tooth law (Fig. 3). The physical scenario that is intended to be simulated and assessed is a temporary obstruction and, therefore, a reduction of the nozzle throat for an imposed period of time. In such a manner, for each selected "shape of the obstruction" in time, a parametric analysis will consider the variation of the peak percentage variation of the throat area $\left(\Delta A_{t} / A_{t}\right)$ and the obstruction duration in time $(\Delta t)$. 


\subsection{Unexpected Increase in Burning Rate/Combustion Surface}

An unexpected propellant burn rate variation or a burning surface sudden modification can be both modeled as an imposed variation of the source terms representing the mass/energy addition from the propellant grain, which combines both the case of an imposed variation of the propellant grain burning rate and the case of a variation of the propellant burning surface.

Consider, in fact, the following expressions (note that the energy source term related to the propellant grain mass addition can be directly obtained from these expressions by using the following equation: $\dot{e}_{p}=\dot{m}_{p} H_{f}$ ):

- variation of the coefficient of the propellant grain APN combustion law ( $\tilde{a}$ is the $a$ perturbed parameter):

$$
\dot{m}_{p}=\rho_{p} S_{b} \tilde{a}\left(\frac{p}{p_{\mathrm{ref}}}\right)^{n}=\rho_{p} S_{b} a\left[1+\delta_{a}(t)\right]\left(\frac{p}{p_{\mathrm{ref}}}\right)^{n} ;
$$

- variation of the exponent of the propellant grain APN combustion law $(\tilde{n}$ is the $n$ perturbed parameter):

$$
\dot{m}_{p}=\rho_{p} S_{b} a\left(\frac{p}{p_{\text {ref }}}\right)^{\tilde{n}}=\rho_{p} S_{b} a\left(\frac{p}{p_{\text {ref }}}\right)^{n\left[1+\delta_{n}(t)\right]} ;
$$

- variation of the propellant burning surface $\left(\tilde{S}_{b}\right.$ is the $S_{b}$ perturbed parameter):

$$
\dot{m}_{p}=\rho_{p} \tilde{S}_{b} a\left(\frac{p}{p_{\mathrm{ref}}}\right)^{n}=\rho_{p} S_{b}\left[1+\delta_{S_{b}}(t)\right] a\left(\frac{p}{p_{\mathrm{ref}}}\right)^{n} .
$$

All the cases can be analyzed considering only the perturbation either of the coefficient, or the exponent of the burning rate law, or of the combustion surface, since the three perturbations are related each other, by equating the expressions for the instantaneous propellant mass addition:

$$
1+\delta_{a}(t)=\frac{p^{n \delta_{n}(t)}}{p_{\mathrm{ref}}} S_{b}=1+\delta_{S_{b}}(t) .
$$

Based on these simple remarks, in the analysis, only the perturbation of the coefficient $a$ of the APN law will be discussed since the variation of the $a$ parameter can be simply translated into the correspondent variation of the $n$ parameter or of the combustion surface $S_{b}$.

Different kinds of imposed variation of the propellant mass addition will be imposed as for the case of the slag ejection simulation: rectangular and triangular increase. 
In particular, it is worth stressing that the unexpected increase of the burning surface area will be considered in the frame of the analysis of the ballistics anomalies, which cause effects only for a small period of time during the firing. For this reason, the possible presence of voids and cracks in propellant grain will be not considered in this work, if not as events which are in some way recovered into the firing of the SRM, in a very small amount of time (i. e., the presence of crack or voids near the burnout of a portion of the propellant grain). Anyhow, the presence of cracks, voids, or debonds can be considered very unlikely events because of the multiple inspections to which the SRM is subjected for its acceptance. Moreover, they represent very simple recognizable cause of an anomaly during the SRM firing, since they imply a modification of the SRM pressure trace for long times into the burn, with a complete drift of the SRM from its nominal behavior.

\section{NUMERICAL SIMULATIONS SETUP: BASELINE SOLID ROCKET MOTOR CONFIGURATION}

The numerical simulations will be carried out for an SRM reference configuration that is an aft-finocyl motor, which geometry is shown in Fig. 4, in terms of port area and burn perimeter distributions along the SRM axis.

The reference SRM configuration is designed as representative of a first solid stage or a solid booster. The SRM is approximatively $10 \mathrm{~m}$ long, with an aft-

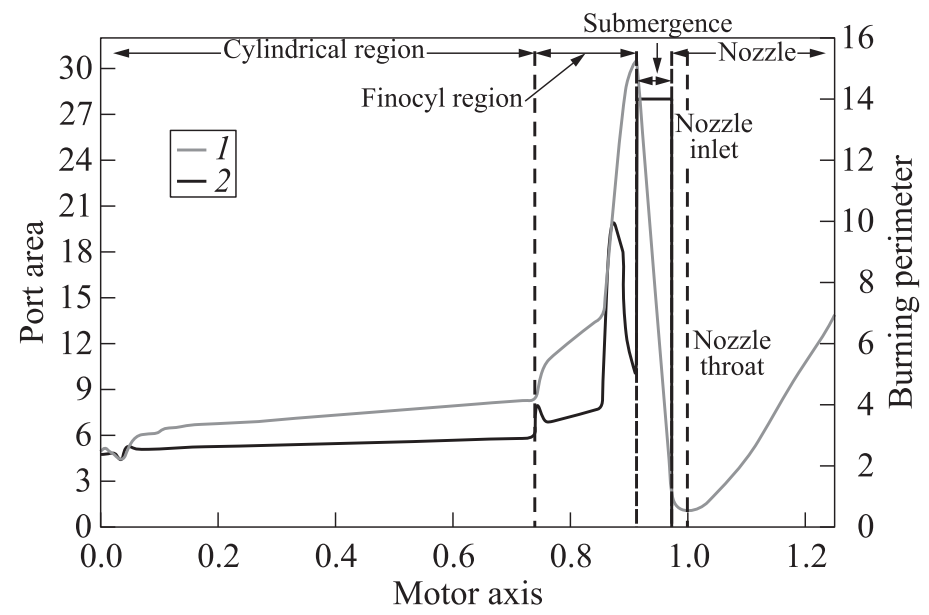

Figure 4 Aft-finocyl QID geometrical configuration: 1 - port area; and 2- burning perimeter 
finocyl geometry similar to all the VEGA solid stages and an $\mathrm{HTPB} / \mathrm{AP} / \mathrm{Al}$ propellant, with an initial propellant mass of around $100 \mathrm{t}$.

The geometrical configuration is designed intentionally to be not representative for the initial geometrical configuration, but rather for a geometrical configuration of interest during the firing of a typical aft-finocyl SRM.

The numerical simulations are performed in a pressure range $67-82$ bar, which is a typical operational range for a first stage/booster, in order to assess the possible effects on the phenomena under analysis of a different operating pressure of the SRM.

A preliminary sensitivity analysis has been performed for the identification of the simulation setup able to provide, with a reasonable precision, consistent numerical results, in the limit of the Q1D unsteady representation of the problem. Therefore, a grid convergence analysis, together with a variation of the order of accuracy of the numerical scheme adopted and of the CFL (Courant-FriedrichsLevy) number has been carried out.

For all the analyzed causes of the ballistic anomalies, a parametric analysis will be performed considering a variation of the model input parameters. For the cases of slag ejection and unexpected increase of burning rate/combustion surface, the parametric analysis will consider a reference pressure of the blip peak amplitude in order to set the input parameters of the perturbations.

All the simulations are made allowing the flowfield to reach its steady-state conditions for the given simulation setup and, then, applying the source of perturbation of the flowfield: the release of an inert object in the flow, or the variation of the nozzle throat area, or the variation of propellant grain mass addition.

\section{RESULTS}

\subsection{Discharge of Inert Object}

A preliminary parametric analysis has been firstly performed for the motion of the particle in the SRM flowfield in order to have sensitivity about the input parameters which play a negligible role in the dynamics of the particle in the bore.

The results have shown that there is almost a negligible effect on the particle dynamics of accounting, even for big sizes of particles, of the gravity and, moreover, that the starting abscissa of the particle influences only the residence time of the particle in the combustion chamber and not its effects on the SRM flowfield. In fact, within the limit of the experimental setup (particle sizes, densities and drag coefficient range of variation) and of the model representation capabilities, the particle motion inside the SRM flowfield is mainly dictated by the abrupt 


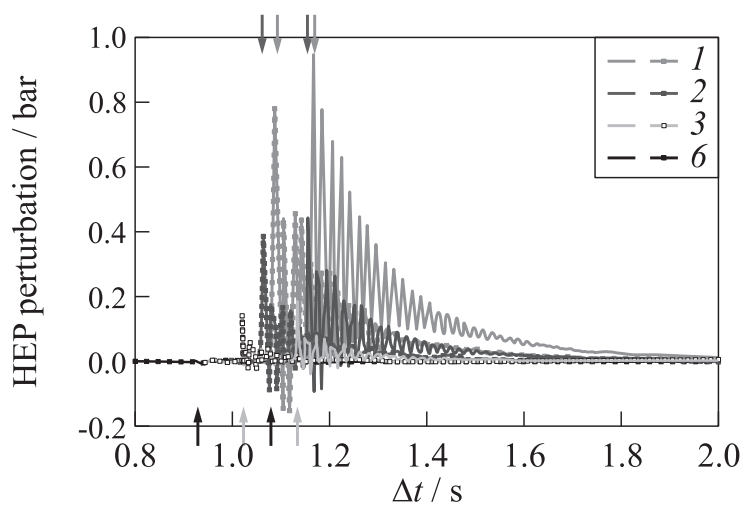

(a)

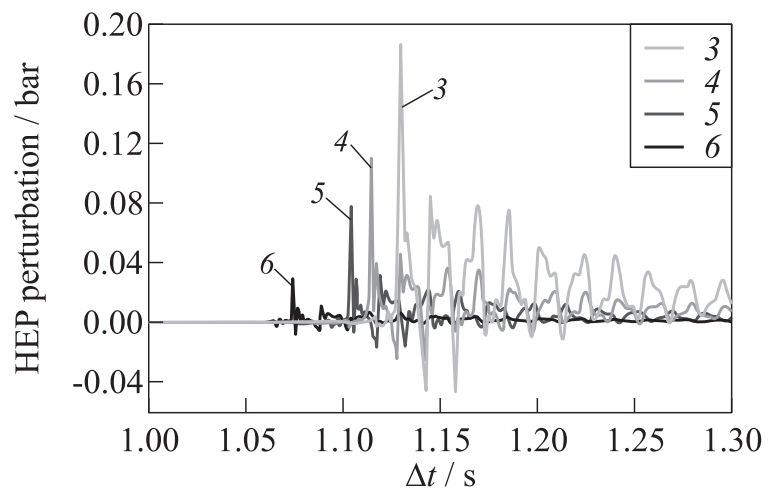

(b)

Figure 5 Particle effects on HEP for different particle radii at $\approx 67$ bar for $\rho_{p}=1900 \mathrm{~kg} / \mathrm{m}^{3}: 1-r_{p}=100 \mathrm{~mm} ; 2-75 ; 3-50 ; 4-40 ; 5-35 ;$ and $6-$ $r_{p}=25 \mathrm{~mm}$. Curves with signs refer to $C_{D}=1$ and without signs to $C_{D}=0.3$

acceleration of the flowfield due to the expansion in the nozzle (which brings about a very intense particle acceleration), where the gravity force is completely negligible in comparison with the drag and, hence, the small variations in the particle velocity at the nozzle entrance section, for different initial conditions, are not so much relevant for the particle dynamics in the nozzle. Instead, far from the nozzle entrance section, the drag and the gravity can be of the same order of magnitude and, hence, the gravity term plays a role in the particle dynamics in this zone (for the acceleration levels of a typical first stage, the same remarks are still valid when considering the SRM acceleration in place of the gravity, in the aforementioned discussion).

Figure $5 a$ shows the HEP perturbation (with respect to the quasi-steady state HEP) for different particle radii and in the range of uncertainty of the 
drag coefficient. The results refer to the selected range of variation for the drag coefficient $\left(C_{D}=0.3\right.$ and 1$)$, for $\rho_{p}=1900 \mathrm{~kg} / \mathrm{m}^{3}$ and for a pressure inside the $\mathrm{SRM}$ of $\approx 67$ bar. Figure $5 b$ depicts the perturbation of the HEP considering the same analysis of Fig. $5 a$, with a refinement of the analysis for different particle radii, for $C_{D}=0.3$ and $\rho_{p}=1900 \mathrm{~kg} / \mathrm{m}^{3}$.

As expected, the higher is the particle radius, the larger is the induced perturbation in the HEP. Moreover, for "small" particle radii $(25-50 \mathrm{~mm})$, the HEP perturbation shows an effect on the SRM internal ballistics that is mainly dictated by an oscillatory transient phenomenon, related to pressure waves traveling back and forth in the chamber, with a variation of the mean pressure in time very low, almost negligible, with respect to the peak value of the pressure perturbation. For "large" particle radii $(75-100 \mathrm{~mm})$, instead, a strong excitement of the first chamber longitudinal mode is present, slightly different from a simple wave propagation phenomenon, which is superimposed with a moderate, but present, variation of the mean pressure level of the chamber.

In all cases, such oscillatory phenomena are damped in some cycle of the chamber characteristic time, up to the recovery of undisturbed conditions of the flowfield. Moreover, it is important to underline that the variation of the drag coefficient of the particle implies only a modification of the first and following peaks amplitude, but not of the qualitative response of the combustion chamber to the particle transit in the flowfield, which depends only upon the particle radius.

The analysis of the flowfield allowed to point out that, in agreement with the references which analyzed similar problems in the literature, the strongest perturbation of the flowfield occurs when the particle transits at the nozzle throat, since that location has both the following characteristics: it is the final section useful for subsonic conditions of the flow that allows pressure perturbations to move upstream and, in the meantime, it is the zone where the flowfield gradients are very intense because of the abrupt expansion of the flow in the nozzle (Fig. 6).

Considering this last remark on the mechanism of perturbation of the flowfield, the following three main parameters can be identified as driving elements of the flowfield perturbation.

The first one is the particle radius, as discussed above, which dictates two different kinds of responses of the combustion chamber: waves propagation, for small particles, and first chamber mode activation superimposed with a shift of the chamber mean pressure, for large particles.

The second one is the particle transit velocity across the nozzle throat, which instead implies a slight variation of the pressure peaks generated in the HEP, without any kind of effect on the chamber qualitative response. In particular, for the same particle radius and SRM operating pressure, the slower is the transit of the particle across the throat, the higher is the amplitude of the pressure wave generated by its passage across the nozzle throat. This fact has been also 


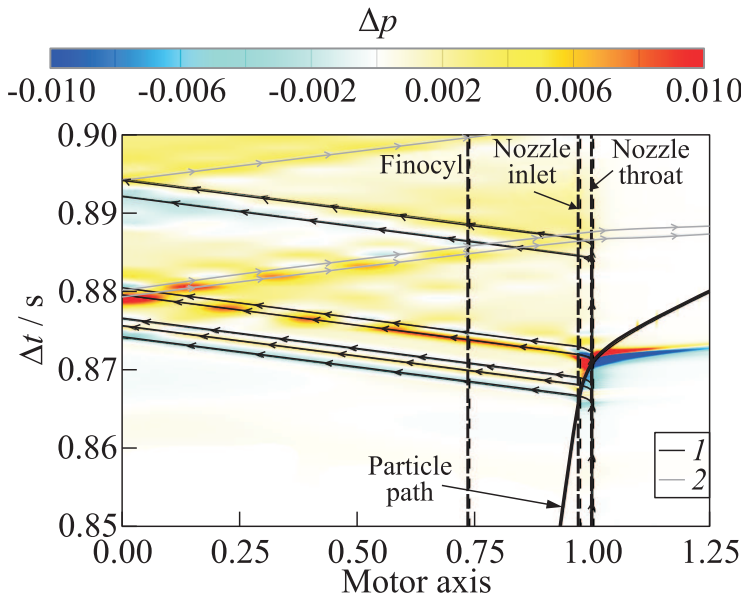

(a)

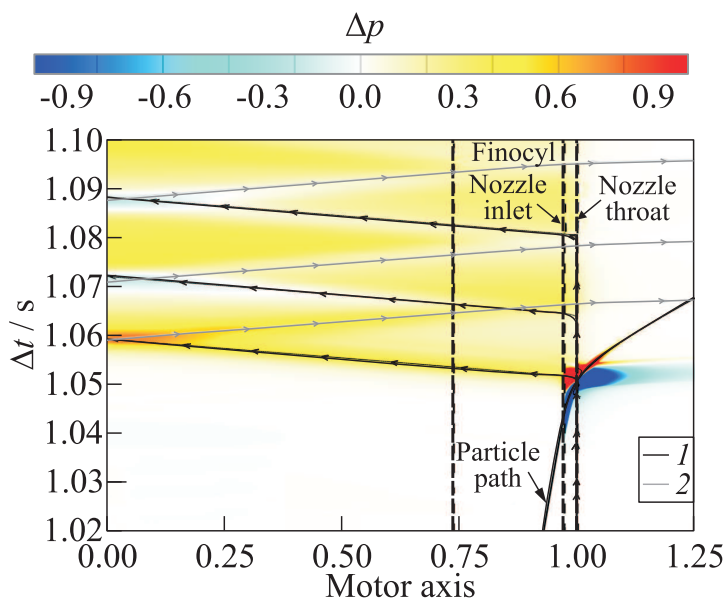

(b)

Figure 6 Pressure perturbation for particles with $r_{p}=25(a)$ and $100 \mathrm{~mm}(b)$, $C_{D}=1$, and $\rho_{p}=1900 \mathrm{~kg} / \mathrm{m}^{3}$ at $p \approx 82$ bar: $1-u-a$; and $2-u+a$

confirmed by the parametric analysis performed for a variation of the following parameters, for a given particle radius: the SRM operating pressure and the particle density. Likewise to the variation of the particle drag coefficient, in fact, both these parameters affect the particle dynamics into the SRM chamber but, in particular, the sudden particle acceleration in the nozzle that, in turn, characterizes the particle transit velocity across the nozzle. Hence, for the same particle size, the flowfield perturbation can be enhanced for a particle with higher 
density and/or for a higher operating pressure of the SRM (and, vice versa, for the opposite cases). In fact, the first one implies directly a lower transit velocity of the particle across the nozzle throat, whereas the second one allows to discuss also the effects of the third parameter which plays a role in the flowfield perturbation: the SRM operating pressure. In fact, a higher operating pressure brings about an increase of the particle transit velocity across the throat, for the other parameters kept constant, but because of the higher level of the SRM operating pressure, a higher perturbation of the SRM head-end occurs with respect to the case with a lower operating pressure. However, if the HEP perturbation is rescaled with respect to the SRM operating pressure, for the same particle size, a higher $\Delta p / p$ is obtained for a particle with higher density or a lower operating pressure.
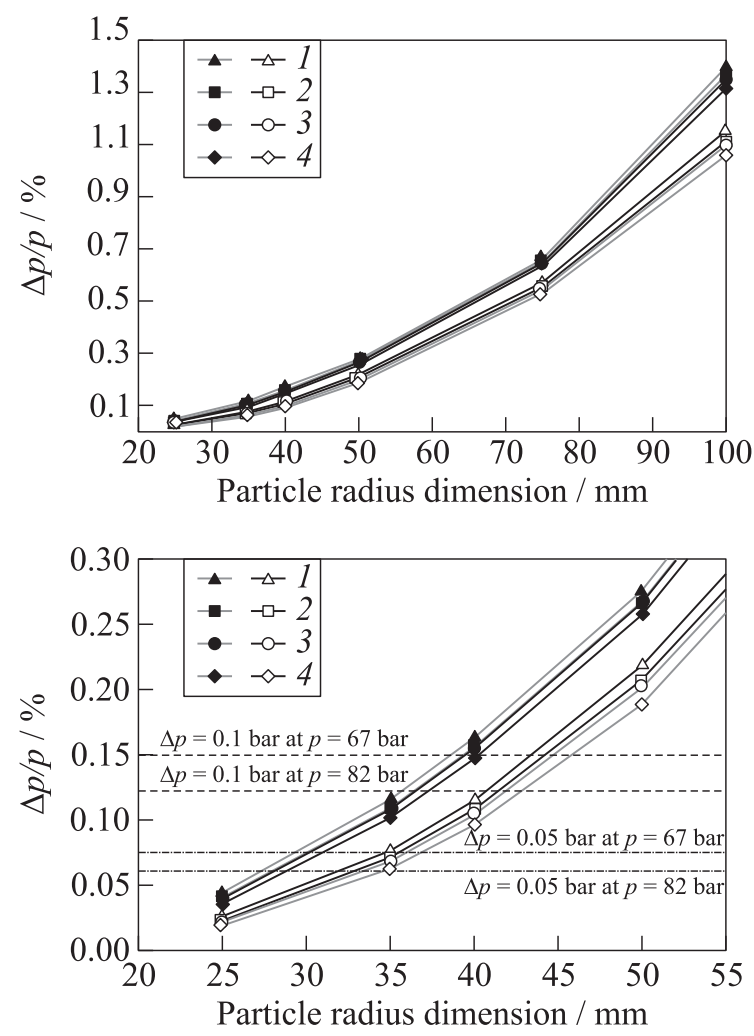

Figure 7 Particle effects on SRM HEP main peak - summary of results: 1 $p=67$ bar and $\rho_{p}=1900 \mathrm{~kg} / \mathrm{m}^{3} ; 2-p=82$ bar and $\rho_{p}=1900 \mathrm{~kg} / \mathrm{m}^{3} ; 3-$ $p=67$ bar and $\rho_{p}=1500 \mathrm{~kg} / \mathrm{m}^{3}$; and $4-p=82$ bar and $\rho_{p}=1500 \mathrm{~kg} / \mathrm{m}^{3}$. Filled signs refer to $C_{D}=0.3$ and empty signs refer to $C_{D}=1$ 
Another parameter, which is investigated and whose effects merits to be discussed, is the shape of the SRM nozzle at the throat. In fact, as discussed before, the source of the main flowfield perturbation is the motion of the particle in a nonuniform flowfield region (the nozzle throat, for the subsonic part, in order to allow the flowfield perturbations to move upstream). Hence, the result of a nozzle shape modification at the throat is that as much the profile of the nozzle is flat, as much it induces less intense gradients of the flowfield around the throat and, in turn, this results in a lower perturbation of the flowfield for the particle transit across the nozzle throat (and, vice versa, for steeper nozzle throat regions).

All the results of the parametric analysis are resumed in Fig. 7 (with the exception of the ones for the nozzle shape modification), where the relationship between the particle size and the peak pressure perturbation at the SRM headend is shown, considering the uncertainties on the particle dynamic in the flow $\left(C_{D}\right)$, for different SRM operating pressures and particle densities analyzed. Figure 7 shows that for a given particle size, there is a small effect of the mean pressure level of the chamber and of the particle density (in the range 1500$\left.1900 \mathrm{~kg} / \mathrm{m}^{3}\right)$, on the resultant percentage variation of the HEP $(\Delta p / p)$. The HEP perturbation, for a given particle dimension, mainly varies because of the uncertainty of the drag coefficient of the particle $\left(C_{D}=0.3-1\right)$. In the selected range of variation of the parameters (particle density, drag coefficient, and mean pressure inside the SRM), Fig. 7 allows assessing the range of particle sizes which are able to provide a given $\Delta p$ at the SRM fore-end (e.g., in order to have a $\Delta p \approx 0.1$ bar, at a range of pressure between 67 and 82 bar, a particle of radius between 36 and $46 \mathrm{~mm}$ has to flow through the nozzle throat).

\subsection{Slag Ejection}

The simulations of the parametric analysis able to assess the effects of a slag ejection phenomenon have been performed considering different time intervals for the nozzle throat blockage $(\Delta t)$ and finding the variation of the throat area blocked $\left(\Delta A_{t} / A_{t}\right)$, which matches the target peak in the HEP perturbation for the different setup conditions considered. The results of the simulations for the rectangular and triangular variation laws and $\Delta p \approx 0.1$ bar at $p \approx 67$ bar are shown in terms of HEP perturbation over time in Figs. 8 and 9 .

Looking at the results, the nozzle throat area variation causes the generation of pressure waves which change the pressure level inside the chamber, but the response of the chamber can be characterized by different phenomena dependently upon the simulation setup. In particular, three different kinds of chamber response can be identified. For $\Delta t / \tau_{a} \ll 1\left(\tau_{a}=1 / f_{a}\right.$ is the chamber first acoustic time), the pressure response is mainly characterized by wave propagation 

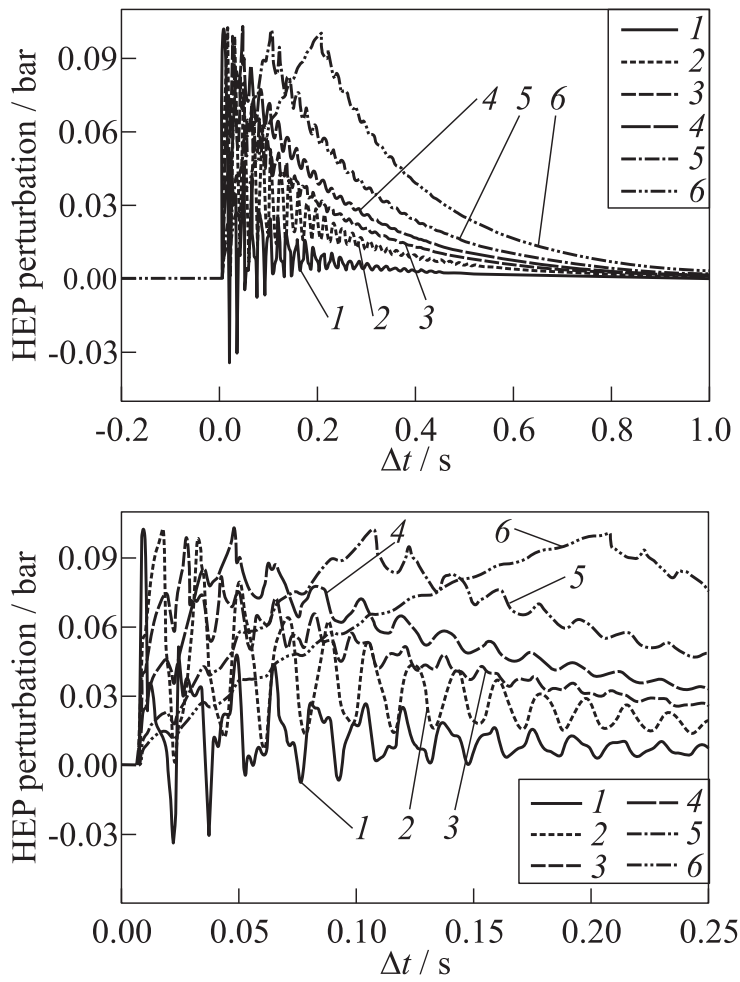

Figure 8 Variation of nozzle throat area with imposed rectangular law, $\Delta p \approx 0.1$ bar at $p \approx 67$ bar: $1-\Delta t=1 \mathrm{~ms}$ and $\Delta A_{t} / A_{t}=2.10 \% ; 2-\Delta t=5 \mathrm{~ms}$ and $\Delta A_{t} / A_{t}$ $=1.15 \% ; 3-\Delta t=10 \mathrm{~ms}$ and $\Delta A_{t} / A_{t}=0.82 \% ; 4-\Delta t=20 \mathrm{~ms}$ and $\Delta A_{t} / A_{t}$ $=0.50 \% ; 5-\Delta t=50 \mathrm{~ms}$ and $\Delta A_{t} / A_{t}=0.25 \%$; and $6-\Delta t=100 \mathrm{~ms}$ and $\Delta A_{t} / A_{t}$ $=0.16 \%$

phenomena, which results in the activation of the first chamber acoustic mode and some more complex wave interaction phenomena, due to the internal shape of the SRM. This region can be further subdivided in two subregions: for very small $\Delta t / \tau_{a}$, the chamber response is characterized by wave propagation phenomena, which do not result in a "clean" activation of the first chamber acoustic mode. Therefore, beyond the chamber first acoustic mode activation, also some more complex wave interactions are present in the HEP trace. The second subregion is, instead, characterized only by an almost "clean" activation of the first chamber acoustic mode.

For $\Delta t / \tau_{a} \gg 1$, the pressure trend is represented by a "bulk response" of the SRM chamber (which could be also adequately characterized with zerodimensional unsteady models). 

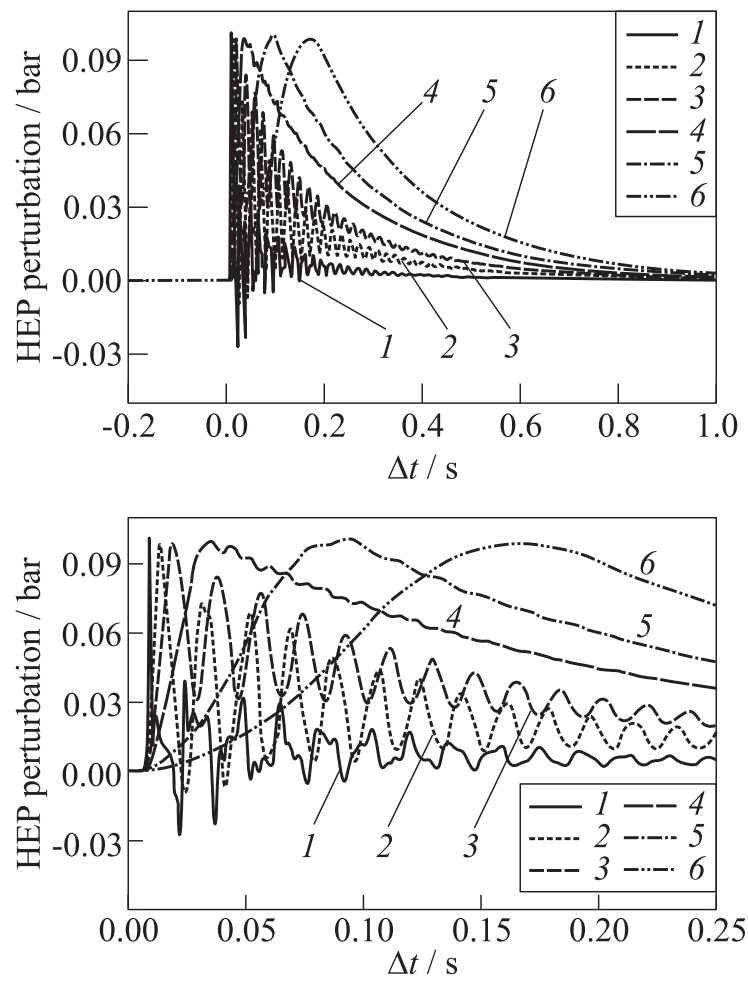

Figure 9 Variation of nozzle throat area with imposed triangular law, $\Delta p \approx 0.1$ bar at $p \approx 67$ bar: $1-\Delta t=1 \mathrm{~ms}$ and $\Delta A_{t} / A_{t}=2.75 \% ; 2-\Delta t=5 \mathrm{~ms}$ and $\Delta A_{t} / A_{t}$ $=1.75 \% ; 3-\Delta t=10 \mathrm{~ms}$ and $\Delta A_{t} / A_{t}=1.40 \% ; 4-\Delta t=20 \mathrm{~ms}$ and $\Delta A_{t} / A_{t}$ $=1.10 \% ; 5-\Delta t=50 \mathrm{~ms}$ and $\Delta A_{t} / A_{t}=0.51 \%$; and $6-\Delta t=100 \mathrm{~ms}$ and $\Delta A_{t} / A_{t}$ $=0.3 \%$

For $\Delta t / \tau_{a} \approx \mathcal{O}(1)$, as border situation, the pressure profile and chamber flowfield are characterized by a combination of a bulk response, with superimposed pressure oscillations due to waves propagation inside the SRM bore, resulting in the activation of the first chamber acoustic mode.

In all cases, as expected, the pressure perturbations need some cycles of the chamber characteristic time in order to recovery undisturbed conditions of the flowfield.

Looking at Fig. 8 or Fig. 9, the case for $\Delta t=1 \mathrm{~ms}$ is similar to the HEP response to the particle motion through the nozzle throat (compare with Fig. $5 b$ ), for a particle radius greater than $25 \mathrm{~mm}$. In fact, in these cases, the time of passage of the particle across the throat is of the order of magnitude of $1 \mathrm{~ms}$, that is, of the same order of magnitude of the imposed variation of the nozzle 
throat. It is worth recalling that for the particle motion inside the SRM flowfield, the most relevant effect on the SRM internal ballistic occurs as the particle moves through the nozzle throat. Therefore, this effect can be modeled as a temporary reduction of the nozzle throat with imposed law, as made in this section. This fact answers to the reason for which the chamber pressure response is similar, when similar forcing terms are imposed to the flowfield.

For a given time of the nozzle throat blockage, the change of the shape of the imposed nozzle throat obstruction (rectangular, triangular, or saw-tooth) changes a little the amount of obstruction necessary to obtain a given HEP perturbation, as well as the borders of the classification of the different kinds of the combustion chamber responses (bulk response, wave propagation response, or a combination of them). In particular, as also qualitatively expected, the border of the distinction among the bulk response and the wave propagation response moves forward, as much as the source of perturbation of the flowfield gets more abrupt (i. e., for the rectangular case) and vice versa. At the same time, for a more abrupt source of perturbation of the flowfield, the peak value of the nozzle throat obstruction necessary to produce the same target HEP peak gets lower for the same time period of the perturbation.

Effect of different solid rocket motor operating pressure and different target $\Delta \boldsymbol{p}$. In order to have some sensitivity on the phenomenon, several other simulations have been performed for the nozzle throat area obstruction simulating a slag ejection phenomenon, considering a variation of the SRM chamber pressure level and of the target peak $\Delta p$ produced at the head-end of the SRM. In this paragraph, a resume of the achieved results will be provided, discussing the effect of a different target $\Delta p \approx 0.5-1$ bar for the same pressure level of 67 bar inside the chamber and analyzing the case of a higher operating pressure of the $\mathrm{SRM}(\approx 82$ bar $)$.

The results have shown that the amplitude of the imposed nozzle throat obstruction, as well as a different SRM operating pressure do not alter at all the classification of different kinds of the combustion chamber response, which is only dependent upon the imposed time interval of the perturbation. Moreover, the amplitude of the throat obstruction is in a linear relationship with the resulting HEP perturbation $(\Delta p)$, whereas for a given nozzle throat obstruction law, the operating pressure acts as a scaling effect of the resultant pressure perturbation, regardless the chamber response regime (wave-dominated or bulk regime).

Hence, for the same SRM operating pressure, $\Delta t$, and shape of the nozzle throat variation, to obtain different peaks in the HEP variation, e. g., $\Delta p \approx 0.5$ 1 bar (small with respect to the HEP of the SRM), a linear relationship stands for the necessary $\Delta A_{t} / A_{t}$ (e.g., if for the rectangular law, $\Delta t=1 \mathrm{~ms}$, the nozzle throat variation necessary to obtain $\Delta p \approx 0.1$ bar is $\Delta A_{t} / A_{t} \approx 2.10 \%$; in order to obtain $\Delta p \approx 0.5$ bar, $\Delta A_{t} / A_{t} \approx 10.5 \%$ is needed, simply five times the $\Delta A_{t} / A_{t}$ necessary to get $\Delta p \approx 0.1$ bar). 
Whereas for different operating pressures of the SRM and for the same $\Delta t$ and shape of the nozzle throat variation, to obtain a given $\Delta p$ (small with respect to the HEP of the SRM), the imposed $\Delta A_{t} / A_{t}$ variation can be assessed considering that a linear rescaling of the imposed perturbation stands, in reason of the ratio of the mean pressure levels in the SRM chamber (e.g., if for the rectangular law, at the $\mathrm{HEP}$ of $\approx 67 \mathrm{bar}, \Delta t=1 \mathrm{~ms}$, the nozzle throat variation necessary to obtain $\Delta p \approx 0.1$ bar is $\Delta A_{t} / A_{t} \approx 2.10 \%$; in order to obtain the same $\Delta p$, at a pressure of $\approx 82$ bar, $\Delta A_{t} / A_{t} \approx 1.733 \%$ is needed, simply $k \approx 67 / 82$ times the $\Delta A_{t} / A_{t}$ necessary to get the same $\Delta p$ at different SRM operating pressure).

Moreover, it is worth stressing that for either the variation of the target peak $\Delta p$, or of the SRM operating pressure, the shape of the HEP over time keeps almost unchanged, since it is only dependent upon the imposed $\Delta t$ and it is only subjected to a variation of the peak value of the resultant $\Delta p$.

These facts allow inferring that in the limit of small $\Delta p$, around the operating pressures analyzed and for a given shape of the nozzle throat obstruction, a linear relationship exists among the imposed peak of percentage variation of the nozzle throat area $\left(\Delta A_{t} / A_{t}\right)$ and the peak value of achieved percentage variation of the HEP $(\Delta p / p)$. The effect of this linear relationship is shown in Fig. 10, which depicts for all the simulations performed the peak values obtained for the SRM HEP against the peak values of the imposed nozzle throat variations. Note that all the curves fit quite well the constraints that for a null variation of the nozzle throat $\left(\Delta A_{t}\right)$, a null variation of pressure perturbation is obtained. This observation coming from the reduction of the data of all the numerical simulations performed allows to extend the validity of the dis-

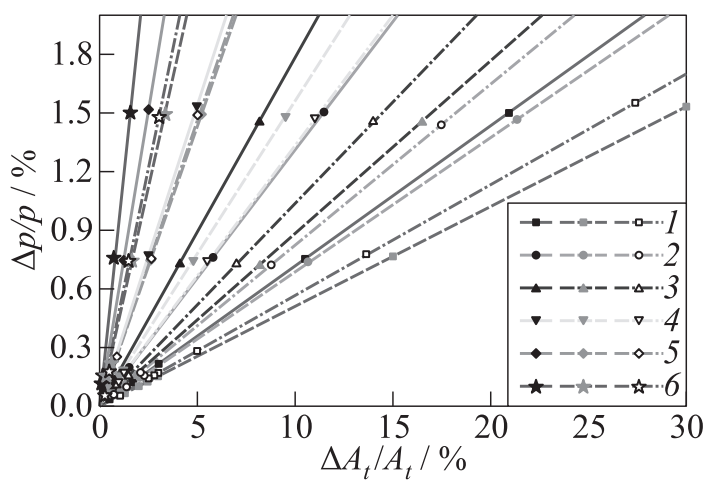

Figure 10 Variation of nozzle throat area with imposed laws: $1-\Delta t=1 \mathrm{~ms}$; $2-5 ; 3-10 ; 4-20 ; 5-50$; and $6-\Delta t=100 \mathrm{~ms}$. Black signs refer to rectangular, grey signs to saw-tooth, and empty signs to triangular nozzle throat laws 
cussed results beyond the cases analyzed, in the limit of small perturbations assumed.

\subsection{Unexpected Increase in Burning Rate / Combustion Surface}

Some preliminary tests have been performed in order to get some sensitivity about the effects on the SRM internal ballistics due to different locations of the imposed variation of the propellant mass addition, modeling an unexpected increase in the burning rate or in the combustion surface. These tests have shown that, excluding very much concentrated impositions of the propellant grain mass addition variation, i. e., very few cells, the results are qualitatively and quantitatively around the ones for the case of an uniform variation of the propellant mass addition on the whole combustion surface, when an appropriate rescaling is considered (e.g., if $\tilde{\Delta}$ is the imposed variation on the whole combustion surface, the case with $N \tilde{\Delta}$ imposed on $1 / N$ of the combustion surface gives the same results, for $N$ small, regardless the location of the perturbation). Therefore, a uniform variation of the propellant grain mass addition has been considered for the simulations discussed in the following.

In case that the variation of the mass addition from the propellant is imposed in a very small region of the combustion surface, the response of the combustion chamber differs a little from the simple scaling rule given above, dependently upon the region location and the percentage of mass addition variation. In fact, both these parameters (location of the region interested in the variation for the mass addition perturbation and percentage of variation of the mass addition) play a role in the response of HEP. In particular, for the same percentage variation of the propellant mass addition, the location of the region interested in the mass addition variation modifies a little the timing at which its effect is felt at the SRM head-end (because of its distance from the SRM fore-end), whereas the percentage variation of the mass addition more (or less) localized in a given region of the combustion surface implies a higher (or lower) perturbation of the flowfield, which, in turn, causes a nonscaling rule for obtaining the given $\Delta p$ that is brought about the nonlinearity of pressure waves generated by the perturbation itself.

Similarly to the case of the imposed variation of the nozzle throat area, the imposed variation of the propellant grain mass addition has two parameters to be set: the time $(\Delta t)$ and the percentage $(\tilde{\Delta})$ of variation of the mass flow rate coming from the propellant grain. As for the case of the variation of the nozzle throat area, the simulations have been performed imposing $\Delta t$ and selecting the percentage variation $\tilde{\Delta}$, which matches a given target $\Delta p$.

The results of the simulations for a target $\Delta p \approx 0.1$ bar are shown in Fig. 11, for the rectangular variation law, and in Fig. 12, for the triangular variation law. Comparing Figs. 11 and 12 with Figs. 8 and 9, the results are qualita- 

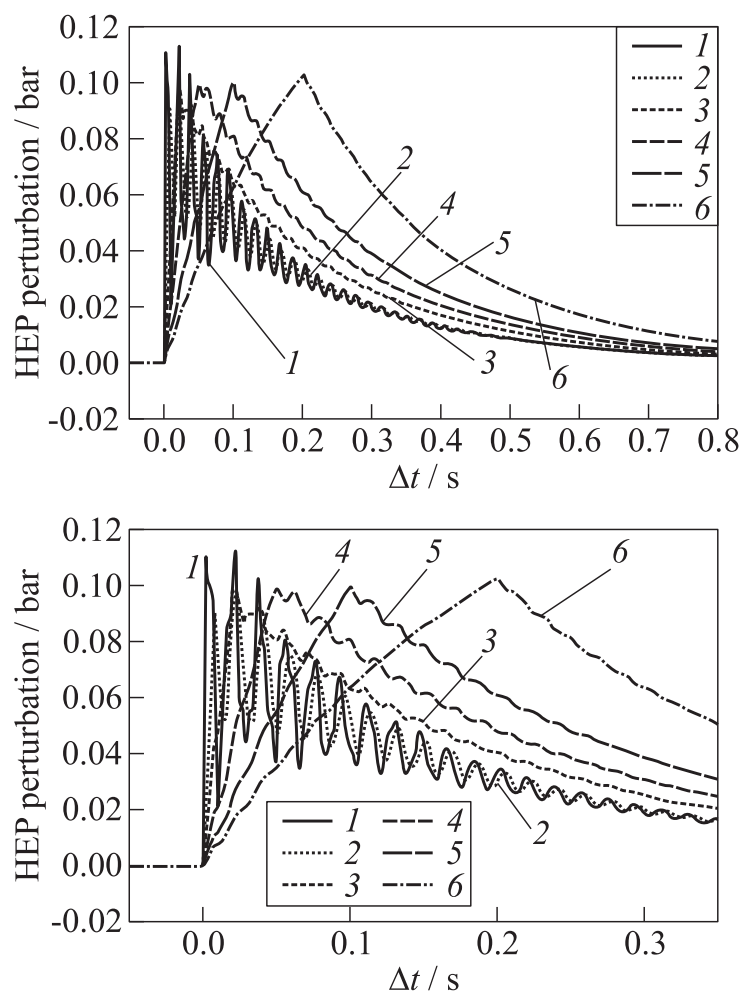

Figure 11 Effect of variation of coefficient of combustion law APN, rectangular perturbation: $1-1 \mathrm{~ms}, 8 \%$; $2-4 \mathrm{~ms}, 2 \%$; $3-10 \mathrm{~ms}, 1 \%$; $4-25 \mathrm{~ms}, 0.45 \%$; $5-$ $50 \mathrm{~ms}, 0.25 \%$; and $6-100 \mathrm{~ms}, 0.16 \%$

tively similar with respect to the ones obtained for the imposition of a nozzle throat area variation (modeling a slag ejection phenomenon), though the forcing on the flowfield is completely different from that case. Really, the differences among the results are ascribable and explainable directly as due to the different kinds of flowfield perturbations imposed in the two different cases. In fact, since the source of the perturbation is less localized for the mass addition perturbation, in comparison with the one of the throat area variation, for the same time window of imposition of the perturbation, the response to the $\dot{m}_{p}$ variation brings about "less organized - more distributed" pressure waves along the chamber than the case of nozzle throat variation. Therefore, for the same $\Delta t$, the result in terms of HEP perturbation is a less oscillatory trend of the HEP, in comparison with the one for the nozzle throat area variation. 

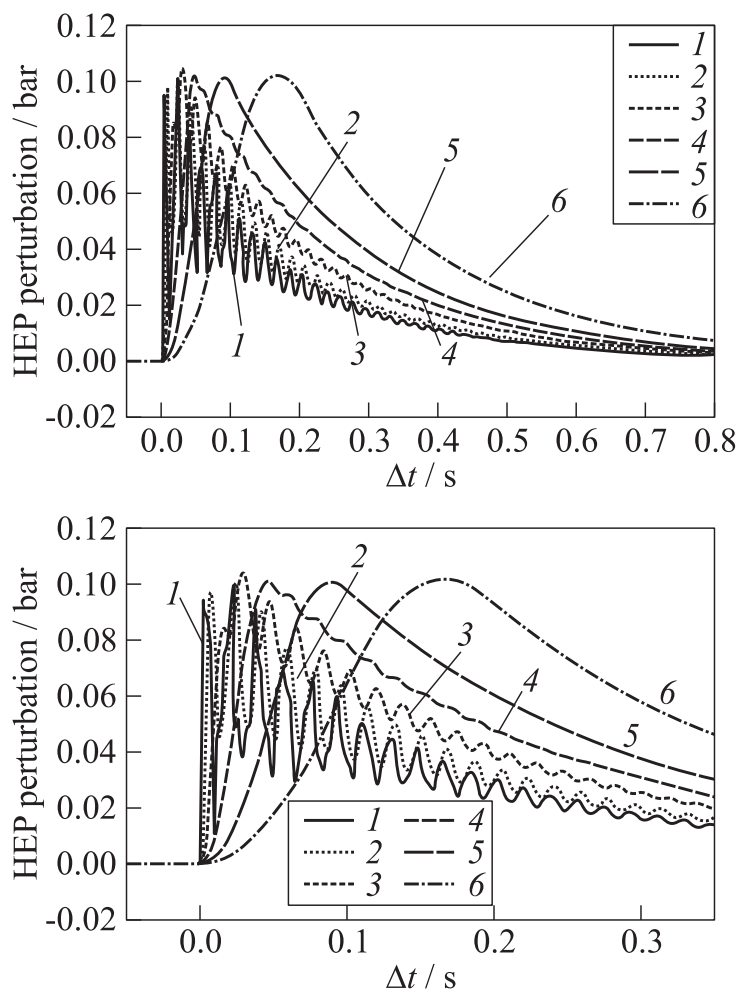

Figure 12 Effect of variation of coefficient of combustion law APN, triangular perturbation: $1-1 \mathrm{~ms}, 14 \% ; 2-4 \mathrm{~ms}, 4 \% ; 3-10 \mathrm{~ms}, 2 \% ; 4-25 \mathrm{~ms}, 0.9 \%$; $5-$ $50 \mathrm{~ms}, 0.5 \%$; and $6-100 \mathrm{~ms}, 0.3 \%$

The main result of this fact is that the classification of the HEP response, on the basis of the time period of the imposed flowfield perturbation in three different regions (bulk response, oscillatory response, or a combination of both) still stands. However, each region limit, characterized in terms of the $\Delta t / \tau_{a}$ parameter, occurs for lower values of this parameter, so that, for example, the subregion characterized only by wave propagation phenomena, not organized in an almost "pure" acoustic excitement of the first chamber mode can be no more detected.

As for the case of the flowfield perturbation due to the nozzle throat variation, a parametric analysis have been performed for different SRM operating pressures and for different target HEP perturbations. The results have confirmed that a scaling rule and a linear relationship among the amplitude of the source and the HEP perturbation stand for, respectively, different operating pressures and different target $\Delta p$, regardless the different regimes of response 


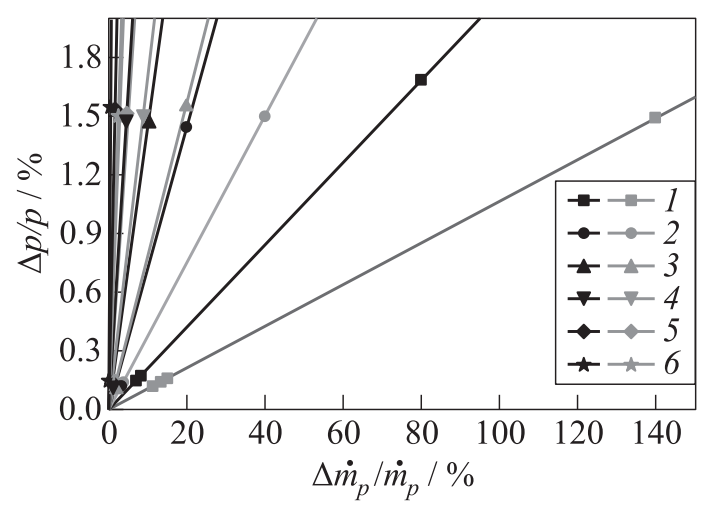

Figure 13 Variation of propellant grain mass addition with imposed laws (black signs - rectangular law and grey signs - triangular law): $1-\Delta t=1 \mathrm{~ms} ; 2-4$; $3-10 ; 4-25 ; 5-50$; and $6-\Delta t=100 \mathrm{~ms}$

of the combustion chamber, which is instead dictated by the $\Delta t / \tau_{a}$ parameter. The results of all the simulations performed are resumed in Fig. 13, where the obtained peaks value of the HEP percentage perturbation are plotted against the variation of the peak of the propellant grain mass addition imposed (for the rectangular and triangular variation laws, different $\Delta t$, and mean pressure of the simulations). Note that as for the case of variation of the nozzle throat area, the straight lines which are fitting the data, fit also with good agreement the trivial solution that for null perturbation, no pressure variation is induced inside the flowfield.

\section{CONCLUDING REMARKS}

The study of different possible causes of ballistic anomalies in SRMs has been performed by means of a wide numerical parametric analysis. The possible sources of investigated ballistics anomalies in SRMs, which bring about unpredicted pressure blips in the SRM pressure traces, are the following: the discharge of an inert object, the slag ejection, and the unexpected increase in the burning rate or in the combustion surface. Each possible cause of the pressure blips has been reasonably modeled in a simplified manner in the framework of a Q1D unsteady model of the SRM internal ballistics. The reference configuration for the study is an aft-finocyl SRM, representative in terms of design of a first stage/small solid booster.

A literature survey has shown the importance of assessing the possible causes of the ballistic anomaly in order to aid in uncovering possible motor design defi- 
ciencies or unique motor characteristics, in real applications where the ballistics anomalies occurred and brought about minor/major anomalies in the SRM operative life, but even catastrophic failures.

The results of the simulations have allowed to acquire a feeling of the physics underlying each cause of the pressure blips. Moreover, and above all, the analysis of the numerical simulations have shown that the ballistics anomalies can be classified by means of the footprint they cause in the HEP of the SRM.

For the motor type under analysis, the ejection of inert objects from the SRM nozzle leads more reasonably to a pressure wave dominated HEP traces, with a very small/negligible variation in the chamber pressure mean value. Only for very big particles, a relevant mean chamber pressure modification superimposed with intense pressure waves, which show the activation of the first chamber acoustic mode, can be observed. The analysis has shown that these two regimes for the combustion chamber response are only dependent upon the particle dimension, whereas the peak values of the pressure oscillations induced at the motor head-end, for a given SRM operating pressure, are mainly dependent upon the velocity of the particle transiting across the nozzle throat. In fact, the main and strongest source of perturbation of the SRM flowfield is represented by the transit of the particle across the nozzle throat, where the flowfield spatial gradients are very intense and the generated disturbances of the flowfield can propagate upstream. Therefore, a modification of the pressure peak due to the particle transit across the nozzle throat can be achieved for a variation of these parameters: the SRM operating pressure (higher operating pressures imply higher pressure fluctuations) and, for the same SRM operating pressure, the nozzle profile around the nozzle throat (a reduction of the pressure peak is obtained for flatter profiles) and the particle transit velocity at the throat (slowest particles bring to higher amplitude pressure fluctuations). In particular, for the same particle size, the pressure perturbation at the motor head-end can be enhanced for a particle with higher density (because of the lower particle transit velocity at the nozzle throat) and/or for a higher operating pressure of the SRM (because of two competitive effects: the direct one of the SRM operating pressure and the one induced by the SRM operating pressure on the particle dynamics which increases the particle velocity at the nozzle throat).

Within the limits of the model used for the slag ejection that is represented as an imposed obstruction of the nozzle throat (as done by other authors in the literature), a similar discussion can be outlined for the slag ejection and the unexpected and sudden increase in the burning rate or in the combustion surface. In fact, in both cases, the HEP trace can be characterized by two different regimes of response and by a transition region, where a combination of the two can be observed: a pressure waves dominated response and a bulk response. These two different regimes for the fore-end pressure response are only dependent upon the ratio of characteristic time period of the distur- 
bance (slag ejection or the increase in the propellant grain mass addition) and the chamber first acoustic mode, regardless the amplitude of the imposed perturbation, which modifies only the amplitude of the resultant pressure peak. Moreover, in both cases, in the limit of the small disturbances considered of the SRM HEP (due to a nozzle temporary obstruction for a slag ejection or a modification of the propellant mass addition), a linear relationship can be observed between the peak of the source of perturbation and the peak of the fore-end pressure obtained. Finally, for the same perturbation cause (nozzle throat obstruction, or propellant grain mass addition), in the limit of the considered cases, the peak of the pressure disturbance at the head-end scales linearly with the operative pressure of the SRM, regardless the fact that the regime of the combustion chamber response is dominated by pressure waves or by a bulk response.

\section{ACKNOWLEDGMENTS}

The authors would like to acknowledge ESA ESRIN Vega Integrated Project Team for the motivation and the support provided to the work.

In memory of Prof. Maurizio Di Giacinto, an outstanding professor, a passionate researcher, a wise mentor, a beloved friend.

\section{REFERENCES}

1. Heister, S. D., and E. M. Landsbaum. 1991. Analysis of ballistic anomalies in solid rocket motors. J. Propul. Power 7(6):887-893. doi: 10.2514/3.23406.

2. Murdock, J. W. 1986. Rocket thrust perturbation from discharge of an inert body. J. Propul. Power 2(2):117-123. doi: 10.2514/3.22854.

3. Whitesides, R. H., and D. C. Purinton. 1995. Effects of slag ejection on solid rocket motor performance. AIAA Paper No. 95-2724. doi: 10.2514/6.1995-2724.

4. Hopson, C. B. 1995. Space shuttle solid rocket motor slag expulsion mechanisms. AIAA Paper No. 95-2725. doi: 10.2514/6.1995-2725.

5. Sambamurthy, J. K., A. Alvarado, and E. C. Mathias. 1996. Correlation of slag expulsion with ballistic anomalies in shuttle solid rocket motors. J. Propul. Power 12(4):625-631. doi: 10.2514/3.24082.

6. Dotson, K.W., J.W. Murdock, and D. K. Kamimoto. 1999. Launch vehicle dynamic and control effects from solid rocket motor slag ejection. J. Propul. Power 15(3):468-475. doi: 10.2514/2.5450.

7. Kibbey, T. P. 2012. One-dimensional, two-phase flow modeling toward interpreting motor slag expulsion phenomena. AIAA Paper No. 2012-3885. doi: 10.2514/6.20123885 . 
8. Blomshield F. S. 2000. Pulsed motor firings. Technical Report NAWCWD TP 8444. China Lake, CA: Naval Air Warfare Center Weapons Division.

9. Lovine, R. L., J. D. Baum, and J. N. Levine. 1985. Ejecta pulsing of subscale solid propellant rocket motors. AIAA J. 23(3):416-423. doi: 10.2514/3.8929.

10. Di Giacinto, M., and F. Serraglia. 2001. Modeling of solid motor start-up. AIAA Paper No. 2001-3448. doi: 10.2514/6.2001-3448.

11. Di Giacinto, M. 2001. Numerical simulation of solid motor ignition transient. Number 5-ISICP-027-8-pp-MDG. Begell House Inc., New York, 2001, June 2002. 5th Symposium (International) on Special Topics in Chemical Propulsion. Stresa, Italy. doi: 10.1615/IntJEnergeticMaterialsChemProp.v5.i1-6.700.

12. Favini, B., F. Serraglia, and M. Di Giacinto. 2002. Modeling of flowfield features during ignition of solid rocket motors. AIAA Paper No.2002-3753. doi: 10.2514/6.2002-3753.

13. Favini, B., M. Di Giacinto, and F. Serraglia. 2003. Acoustic phenomena during SRM ignition transient. 10th Workshop (International) on Combustion and Propulsion. Lerici, La Spezia, Italy.

14. Cavallini, E., B. Favini, M. Di Giacinto, and J. Steelant. 2011. A simplified coupled structural-flowfield analysis of solid rocket motors ignition transient. 7th European Symposium on Aerothermodynamics for Space Vehicles Proceedings. Brugge, Belgium. ESA SP-692. 107.

15. Loth, E. 2008. Compressibility and rarefaction effects on drag of a spherical particle. AIAA J. 46(9):2219-2228. doi: 10.2514/1.28943.

16. Clift, R., and W.H. Gauvin. 1970. CHEMECA 70 Proceedings. Butterworth, Melbourne. 1:14-28.

17. Salita, M. 1995. Deficiencies and requirements in modeling of slag generation in solid rocket motors. J. Propul. Power 11(1):10-23. doi: 10.2514/3.23835. 